\title{
Solving problems by analogy: The benefits and detriments of hints and depressed moods
}

\author{
PAULA T. HERTEL and ALICIA J. KNOEDLER \\ Trinity University, San Antonio, Texas
}

\begin{abstract}
In Experiment 1, mildly depressed (dysphoric) and nondysphoric subjects tried to solve logic problems that were analogous to subsequent target problems; then they attempted target solutions with or without hints in the form of the anologues' themes. Target solutions were impaired by the hints in the nondysphoric group alone. Experiment $2 \mathrm{~A}$ was a no-training control to verify that transfer did indeed occur. In Experiment 2B, all subjects received hints in the transfer phase; the training phase was either problem oriented (as in Experiment 1) or memory oriented. Again, nondysphoric subjects solved fewer problems following problem-oriented training than did both dysphoric subjects in that condition and nondysphoric subjects with memory-oriented training. Experiment 3 replicated the previous results in the nondysphoric samples. We interpret these findings within the transferappropriate processing framework.
\end{abstract}

The domain of this research is problem solving by analogy to similar prior experience. Against a tradition that has mainly shown poor transfer of solution methods when hints to use prior analogues have not been provided, a few studies point to the importance of transferappropriate processing (e.g., Adams et al., 1988; Gick \& McGarry, 1992; Lockhart, Lamon, \& Gick, 1988; Stein, Way, Benningfield, \& Hedgecough, 1986). Consider a set of experiments by Needham and Begg (1991): Some of their subjects tried to solve training analogues before they were taught their solutions, while the others studied the analogues and tried to remember them before the solutions were taught. Those who had tried to solve (mostly unsuccessfully) showed superior spontaneous (no-hint) transfer of the methods of solution to subsequent target problems. In the spirit of transfer-appropriate processing (Morris, Bransford, \& Franks, 1977), attempting to solve on both occasions was more beneficial than attempting to remember on the first occasion and attempting to solve on the second.

The specific focus of the present experiments was the nature of the memory component of transfer in Needham and Begg's (1991) paradigm. We considered two possible ways in which memory for the training analogues might influence performance in the transfer phase: (1) Subjects might attempt deliberately to recall training problems

A portion of Experiment 1 was conducted by the second author in partial fulfillment of Honors in Psychology. Experiment 2B was partly supported by the Hogg Foundation for Mental Health; manuscript preparation was supported by a Trinity University Summer Stipend to the first author. We are grateful to Mariana Garza, Kevin Gluck, Laurie Grams, Vickie Schafer, and Christine Winston for their service as experimenters and scorers. Robert Greene and two anonymous reviewers provided very helpful comments on a previous version of the article. Please address correspondence to P. T. Hertel, Department of Psychology, Trinity University, 715 Stadium Drive, San Antonio, TX 78212 (e-mail: phertel@trinity.edu) with similar structural features to arrive at their solutions, or (2) the training analogues and their solutions might come to mind more "spontaneously" as subjects were reminded by the analogous structures of the target problems (see Ross \& Kennedy, 1990). The first waydeliberate recall of analogues-would be less consistent with the transfer-appropriate processing framework invoked by Needham and Begg to explain their findings; attempts to remember should help memory-oriented subjects at least as well as problem-oriented subjects.

In our first attempt to investigate the memory component of transfer following problem-oriented training, we examined the effect of providing hints to recall the analogue. Immediately before each target problem was revealed, some subjects were given an explicit hint in the form of the analogue's title or theme; the others were told to relax and clear their minds. If hints improved performance, we would know that deliberate recall of the analogue could at least facilitate transfer within this paradigm and might even characterize transfer in the absence of such hints (assuming that the appropriate analogues would not always be recalled without them).

To address the possibility that transfer in the absence of hints might be based in part on deliberate recall of the analogues, we broadened the domain of this research to include dysphoric subjects (subjects who feel depressed, but are not formally diagnosed as such). If Needham and Begg's (1991) subjects (who we assume were mainly nondysphoric) explicitly thought back to the training phase to recall problems with similar structures, we should expect dysphoric subjects to show poor transfer in the nohint condition. Dysphoric and depressed subjects have shown a lack of initiative in monitoring the relevance of the past when they are not instructed to do so (see Hertel \& Hardin, 1990). However, the dysphoric subjects should show improved performance in the hint condition, in which the dependence on initiative is reduced by di- 
rections to the appropriate analogy. The latter prediction rests on evidence that dysphoric subjects benefit from instructions to monitor the past (Hertel \& Hardin, 1990). Whether the improvement is sufficient to remove the impairment would depend on whether or not mood affects other components of problem solving (e.g., mapping the structure of the analogue onto the target problem and adapting the solution). If, on the other hand, the performance of dysphoric subjects in the no-hint condition were not impaired, we would have some reason to suspect that initiative in recalling the analogues does not play a large role in no-hint transfer, regardless of the outcome following hints.

Finally, the inclusion of dysphoric groups also suggested a third issue and corresponding factor in the design. The background for this issue was Needham and Begg's (1991) suggestion that spontaneous transfer depends on whether the analogues, rather than other thoughts, come to mind. Task-irrelevant and self-relevant thoughts seem to intrude when depressed, dysphoric, or sadness-induced subjects perform cognitive tasks (e.g., Ingram, 1990; Seibert \& Ellis, 1991). Therefore, we varied the emotional tone of the target problems as a way of determining whether retrieval of the analogue might be impaired by irrelevant thoughts. One third of the subjects attempted to solve the targets from Needham and Begg (1991); the others saw one of two elaborated versions. These versions were constructed around each nonelaborated problem by adding material that was either neutral or depressing (in our opinion). Although the neutral elaborated version was necessary on the grounds of unconfounding problem length with emotional tone, it was also useful for investigating whether dysphoric subjects might be diverted simply by additional material regardless of its relevance to their concerns. Furthermore, the combination of this manipulation with the manipulation of instruction allowed us to discover whether the provision of hints might compensate for the effects of potential distractions on problem solving by analogy.

\section{EXPERIMENT 1}

The subjects in Experiment 1 were categorized as dysphoric or nondysphoric on the basis of responses to mood questionnaires. Within these groups, they were randomly assigned, under the constraint of equal cell sizes, to solve target problems with or without hints regarding the appropriate analogues and to receive targets that were either simple, elaborated with neutral material, or elaborated with depressing material. These two factors were crossed and manipulated between subjects. Everyone received problem-oriented training.

Method
Materials
The subjects' materials consisted of two packets of logic or in-
sight problems. The first packet contained 6 problems: the 5 train-
ing problems from Needham and Begg $(1991)$ and 1 filler from

Phillips (1961). The second packet contained 11 problems: the 5 target problems from Needham and Begg and 6 filler problems from Phillips (1961) and Mott-Smith (1954). The target problems were analogous to the training problems, but the fillers were dissimilar in structural and surface features. Fillers were added as distractors, because we were concerned about possible ceiling effects in using the materials from Needham and Begg alone. Each problem in both packets was typed on the top portion of a separate page, with the bottom reserved for the attempt at solution; no titles were used. The second packet also contained 11 instructional pages, each placed immediately before a problem. The appendix contains an example of a training problem and its corresponding target problem (in three versions). In keeping with Needham and Begg's procedure, the training problems and target problems were maintained in those categories for all subjects; no counterbalancing was used.

Training packet. The presentation order in the training packet was as follows: Poker Chips, Jungle Explorer, filler, T-shirt Vendor, Game-show Contestant, and Baseball Probability. This order was arbitrarily selected on the basis of Needham and Begg's findings of no order effects.

Target packet. The target problems in this set were presented in the same order as their analogues in the training packet, with fillers interspersed. Specifically, the order was: two fillers, Apples and Oranges, filler, Two-string, filler, Poisoned Cups, filler, Liar/ Truth-teller, filler, Probability.

Target problems were presented in one of three versions. The simple versions were copied from Needham and Begg, with the exception of a very few minor changes in wording. The elaborated versions contained additional phrases and sentences that gave the problems either a neutral tone or a depressing tone. Although these additions differed in length across the five targets, the length was approximately the same for both elaborated versions of each. For example, the simple version of Apples and Oranges contained 92 words; it described a grocer in a situation of unscrambling mislabeled boxes. The neutral elaborated version contained 184 words that created a background story of details about opening the store and stocking shelves; the depressing version ( 182 words) provided background about the grocer's financial trouble - his only relief would come from betting on his ability to rectify the mislabeling. The average number of words added to the simple versions to make the neutral and depressing versions was 122 and 120 , respectively.

Instructions in the target packet. Preceding each filler problem in the target packet was this instruction: "Please take this time to clear your mind, take a deep breath, and relax. The experimenter will indicate when it is time to continue." This instruction was typed on a separate page and also preceded every target problem in the no-hint condition. In contrast, instructional sheets preceding targets in the hint condition contained explicit instructions to think back to a previously learned, analogous training problem. For example, for the problems presented in the appendix, this sheet stated:

The Jungle Explorer problem you saw earlier is similar to the problem you are about to solve. Try to use the Jungle Explorer problem to help you solve this problem. In particular, try to use what you learned about how to solve the Jungle Explorer problem to come up with the same kind of procedure for solving this problem. There may be other ways to solve this problem, but it is very important that you try to use what you learned from the Jungle Explorer problem to help you solve this problem.

These instructions were adapted from a similar instruction used by Novick and Holyoak (1991).

Questionnaires. The questionnaires included the Beck Depression Inventory (BDI; Beck, Ward, Mendelson, Mock, \& Erbaugh, 1961) and a questionnaire of our own invention. The BDI is a standard inventory for revealing the syndrome of depression (not its formal diagnosis); college students who score between 9 and 16 should be considered dysphoric, whereas those who score 
Table 1

Measures of Subject Characteristics in Experiments 1, 2A, and 2B

\begin{tabular}{lccccc}
\hline & \multicolumn{2}{c}{ F/M Ratio } & & \multicolumn{2}{c}{ Mean BDI } \\
\cline { 2 - 2 } \cline { 5 - 6 } & Nondysphoric & Dysphoric & & Nondysphoric & Dysphoric \\
\hline Experiment 1: Problem oriented & & & & & \\
$\quad$ No hints & $27 / 21$ & $23 / 13$ & & 3.3 & 15.9 \\
$\quad$ Hints & $28 / 20$ & $21 / 15$ & & 3.6 & 15.7 \\
Experiment 2A: No training & $9 / 9$ & $9 / 7$ & & 4.0 & 16.2 \\
Experiment 2B: Hints & $19 / 9$ & $11 / 9$ & & 4.0 & 14.6 \\
$\quad$ Problem oriented & $15 / 13$ & $16 / 4$ & 3.6 & 14.8 \\
$\quad$ Memory oriented & & &
\end{tabular}

Note $-\mathrm{F} / \mathrm{M}$ ratio $=$ the number of female to the number of male subjects, collapsed across groups receiving different types of target problems.

higher might be considered depressed if they meet certain criteria on a structured, clinical interview (Kendall, Hollon, Beck, Hammen, \& Ingram, 1987). The second questionnaire, which posed questions about the subject's health and emotional well-being, was used to verify the dysphoric states of subjects and to rule out conditions related to drug use or sickness.

\section{Subjects and Design}

Screening. We administered the BDI in lower division psychology classes at Trinity University. In that setting, students filled out the BDI anonymously, but signed a consent form that was coded with a subject number that matched the BDI code. An experimenter then prepared lists of names, phone numbers, and codes from the stack of consent forms. A separate list of BDI scores and codes provided the basis for assigning codes to experimental conditions. We attempted to recruit the subjects with the highest BDI scores, but selected others randomly and assigned them randomly to experimental conditions (under the constraint of equal cell sizes). The experimenters received the assignments and attempted to recruit the students to participate in an experiment on problem solving. The experimenters were therefore blind to mood categories, and the subjects believed that they were being recruited merely through their membership in the departmental phone pool. A total of 174 students participated in the experiment; they had filled out the BDI from 7 to 62 days $(M=27)$ prior to participation.

Final sample. The data from 6 subjects were set aside because their end-of-session BDI scores or responses on the questionnaire placed them outside the categories established for the two mood groups. Subjects in the dysphoric group scored 9 or above on the $\mathrm{BDI}$ on both administrations, or merely on the last administration accompanied by a questionnaire report of having been in a depressed mood for at least 2 weeks. Subjects in the nondysphoric group scored 9 or below on the last BDI and reported the absence of a depressed mood on the questionnaire. The final sample included 16 nondysphoric subjects and 12 dysphoric subjects in each combination of instructions (hint vs. no hint) and type of target (simple, neutral elaborated, or depressing). Table 1 reports the number of women and men assigned to each condition of instruction and mood group, along with their mean score on the BDI at the end of the session. Other than the factor for mood group, no effect in the analysis of BDI scores was reliable $(F \mathrm{~s}<1.0)$.

\section{Procedure}

The subjects were assigned to experimental sessions in groups of 1 to 4 ; the sessions differed systematically only according to whether simple or elaborated targets were used. In each session, the subjects were initially told that they would receive a series of problems to solve. Packets of training problems were distributed, and the subjects were told not to turn the page until they were instructed to do so. They were warned that the problems were difficult and the pace was fast but that it was, nevertheless, very important that they try to solve the problem. The time allotted for each of the training problems was $2.5 \mathrm{~min}$, after which the experimenter solved the problem and explained the solution. (Explanations were taken from Needham and Begg, 1991). Each explanation took approximately $2.5 \mathrm{~min}$ to read, after which the subjects were instructed to turn the page and begin the next problem.

Next, the experimenter distributed the target packets and told the subjects that they contained a different set of problems to solve. The subjects were also told that there were time limits for reading instruction pages and for solving problems. The experimenter announced the time to turn the page. The allotted time for each instruction page was $15 \mathrm{sec}$; the allotted time for the problems differed according to the type of target. The subjects assigned to the simple problems were given $2.5 \mathrm{~min}$ to read and solve each of the 11 problems in the packet; the subjects assigned to the elaborate problems were given $2.5 \mathrm{~min}$ for each filler and $3.0 \mathrm{~min}$ for each target. (Before the experiment, volunteers had been timed for the reading of each problem, and the simple and elaborate problems had required, respectively, approximately 30 and $60 \mathrm{sec}$.) Solutions were not provided by the experimenter. At the end of the session, the subjects filled out the BDI and questionnaire (containing subject codes but no names) and sealed them in an envelope. The experimenter explained that she or he would not see those forms.

\section{Scoring}

Attempts to solve the target problems were scored independently by 2 judges, who were blind to the type of instruction (hint vs. no hint). They agreed on $90 \%$ of their judgments; when they did not, they collaborated to make final decisions. Solutions to each target problem were scored as incorrect or correct, according to criteria established by extracting key statements from the experimenter-provided solutions to the training problems (see the example in the appendix.) The judges did not score solutions as correct if they merely appeared to be "on the right track." The dependent variable for the main analysis was the percentage of correct solutions, out of the possible five. The percentages of training problems solved were scored similarly by 1 judge alone.

\section{Results and Discussion}

\section{Percentage of Targets Solved}

The percentages solved correctly were submitted to an analysis of variance with between-subjects factors for mood (dysphoric or nondysphoric), instruction (hint vs. no hint), and type of target (simple, neutral, or depressing). Table 2 presents those means. We used a significance level of .05 for hypothesis testing and do not routinely report reliable main effects that are qualified by reliable interactions. (A separate analysis failed to reveal reliable effects associated with gender.) 
Table 2

Mean Percentage of Targets Solved After Problem-Oriented Training (Experiment 1)

\begin{tabular}{lcccc}
\hline $\begin{array}{c}\text { Target } \\
\text { Instructions }\end{array}$ & \multicolumn{4}{c}{ Type of Targets } \\
\cline { 3 - 5 } & Simple & Neutral & Depressing & Overall \\
\hline $\begin{array}{l}\text { No Hints } \\
\quad \text { Nondysphoric }\end{array}$ & 68.8 & 67.5 & 76.2 & 70.9 \\
$\begin{array}{l}\text { Dysphoric } \\
\text { Hints }\end{array}$ & 65.0 & 63.3 & 65.0 & 64.4 \\
$\quad$ Nondysphoric & 50.0 & 56.2 & 56.2 & 54.2 \\
$\quad$ Dysphoric & 70.0 & 75.0 & 63.3 & 69.4 \\
\hline
\end{tabular}

Note $-n=16$ in nondysphoric conditions and 12 in dysphoric conditions.

The only reliable effect in the overall design was the interaction of $\operatorname{mood}$ with instruction $[F(1,156)=9.51$, $\left.M S_{\mathrm{e}}=507.91\right] .{ }^{1}$ To our surprise, nondysphoric subjects solved more problems when they were not given hints for the analogies $[71 \%$ vs. $54 \%$ in the hint condition; $F(1,156)=13.13]$. These results suggest that transfer in the no-hint condition was achieved mainly through remindings provided by the targets rather than by a deliberate search of memory for the training analogue. If the latter basis had been predominant, nondysphoric subjects in the no-hint condition would not have solved more target problems than did those to whom we gave explicit hints. Instead, the provision of thematic hints seemed to have disturbed the process of reminding during attempts to solve the targets, perhaps by focusing efforts on remembering details of the training analogues that were irrelevant to their use or by cuing incorrect solutions.

Consistent with the interpretation that spontaneous reminding characterized transfer in the no-hint condition, we did not find a reliable impairment in the dysphoric group, although a trend in that direction was found [ $64 \%$ in the dysphoric group vs. $71 \%$ in the nondysphoric group; $F(1,156)=2.13$ ].

The most interesting view of the interaction of mood with instructions emphasizes performance by the dysphoric subjects who were given hints. Although their performance was similar to that of the dysphoric subjects in the no-hint condition (69\% vs. $64 \%$, respectively, $F<$ 1.0 ), these subjects solved more targets than did the nondysphoric subjects who were given hints $[69 \%$ vs. $54 \% ; F(1,156)=10.62]$. On a post hoc basis, we suggest that the dysphoric subjects failed to concentrate on remembering the prior analogues (and thereby did not experience the disadvantage shown by nondysphoric subjects). Such efforts might require more self-sustained attention than dysphoric subjects can or will muster (see Hertel \& Rude, 1991). A replication of this depressionrelated advantage in the context of thematic hints was one goal of Experiment 2B.

Finally, all effects involving the factor for type of target were unreliable $(F \mathrm{~s}<1.0)$. The remaining experiments also included this factor and failed to reveal reliable effects. This consistent failure could result from the relatively low level of depression experienced by our subjects or from a weak manipulation of problem content.

\section{Number of Incomplete Solutions}

Perhaps the disadvantage of hints for nondysphoric subjects resulted merely from the strict time limits. This might occur, for example, if these subjects continued their efforts to recall the training problem during the time allotted for target solution and ran out of time in applying the method. We therefore evaluated the data for the number of incomplete attempts to solve the target problems, independent of solution accuracy. Criteria included one or more of the following: (1) the last phrase required more words to make sense, (2) the last word was incomplete, (3) a calculation was incomplete, and (4) the page was left blank. The average number of incomplete solutions, out of 5 , was 1.4 ; it did not reliably differ according to mood or experimental conditions $(F \mathrm{~s}<1.5)$.

\section{Percentage of Training Problems Solved}

Dysphoric subjects solved more of the training problems, on average, than did the nondysphoric subjects ( $8 \%$ vs. $5 \%$, respectively), although neither this difference $\left[F(1,166)=2.02, M S_{\mathrm{e}}=127.41\right]$ nor other effects were reliable. Furthermore, although these percentages were correlated with the percentages of targets solved $\left[\beta=.29 ; F(1,155)=14.40, M S_{\mathrm{e}}=467.74\right]$, an analysis of covariance again revealed the reliable interaction of mood with instruction $[F(1,155)=11.71]$.

\section{EXPERIMENTS 2A AND 2B}

Needham and Begg (1991) found that problemoriented training was better than memory-oriented training in facilitating spontaneous transfer. Their memoryoriented subjects, who were trained to remember the first set of problems for a later test, solved fewer problems than did problem-oriented subjects, presumably because the memory orientation in the training phase did not match the problem orientation in the transfer phase. By similar reasoning, we can interpret the surprising results from the nondysphoric groups in Experiment 1 to be consistent with the framework of transfer-appropriate processing: The memory orientation induced by the provision of thematic hints was inconsistent with the problem orientation during the training phase, in which encounters with the analogues were not aimed toward establishing routes for deliberate retrieval. Moreover, this interpretation leads to the prediction that memoryoriented training would be superior to problem-oriented training when explicit hints are subsequently provided. Memory-oriented training should provide a better basis for the use of thematic hints to recall the analogues. In Experiment 2B, therefore, we compared performance in the two training conditions when the hints were given to everyone. This design also allowed us to replicate the other surprising outcome of Experiment 1: the dysphoric advantage in the hint condition.

Experiment $2 \mathrm{~A}$ was conducted separately to provide a baseline comparison for ensuring that transfer indeed occurred in the experimental conditions. These subjects did not receive a pseudotraining phase because we were 
concerned that so little success in solving (without being taught solutions) would discourage them in the second phase and deflate the level of performance below what it would otherwise be. Instead, they were given the second packet of 11 problems immediately, separated by 15 -sec rest intervals, as in the no-hint conditions of Experiment 1.

The designs of Experiments $2 \mathrm{~A}$ and $2 \mathrm{~B}$ again included a variation in the type of target problems, but the simple problems were eliminated, inasmuch as the results of Experiment 1 had failed to suggest that performance on those problems was superior to that on the neutral elaborations. We therefore used the neutral and depressing sets in Experiments 2A and 2B in continuing to investigate the possibly distracting effects of mood-related materials.

\section{Method}

\section{Subjects and Design}

Experiment 2A. Screening procedures and mood criteria described in Experiment 1 were used to recruit 34 participants; 9 nondysphoric subjects and 8 dysphoric subjects received packets with neutral target problems, and 9 nondysphoric and 8 dysphoric subjects received depressing target problems. Packets were assigned randomly within mood groups, with the constraint of equal numbers for each type of target. Subjects participated in groups of 4 to 8 an average of 14 days after the initial BDI score was taken.

Experiment 2B. Similarly, we recruited 102 participants for this experiment. (The data from 6 were set aside, according to mood criteria.) The final sample contained 56 nondysphoric and 40 dysphoric subjects, who were assigned to the factorial combination of orientation during training (problem-oriented vs. memoryoriented) and type of target (neutral vs. depressing). Assignment to experimental conditions was random, under the constraint of equal cell sizes within each mood condition. The subjects participated an average of 19 days after class administration of the BDI. They participated in groups of 1 to 4 in sessions that varied according to training orientation.

Subject characteristics. Table 1 includes the ratio of number of female to male subjects, collapsed across type of targets, and the mean BDI scores at the end of the session. In both experiments, the BDI scores did not vary reliably according to factors in the designs other than mood group, and interactions were not reliable $\left(F_{\mathrm{S}}<1.2\right)$.

\section{Materials and Procedure}

In Experiment $2 \mathrm{~A}$, the materials and procedure were taken directly from the no-hint condition of the transfer phase of Experiment 1 . In the problem-oriented sessions of Experiment 2B, the materials and procedures conformed to those used in the hint condition of Experiment 1. Memory-oriented sessions required the following changes. First, instructions for the first packet of training problems were:

This package contains a set of stories that describe problems. Please read each story very carefully because you will be asked to remember it later. Other subjects in this experiment will work on trying to solve the problems. You are in a control group because we want to know how well the problems can be remembered-not solved. After you study each story, I will explain the solution. It is important that you try to remember these stories and their solutions and that you not try to solve the problems.

Second, the instructional pages preceding each target problem told subjects to use what they remembered from the prior (specified) analogue as they tried to solve the upcoming problem.

Attempts to solve target problems in Experiment 2B were evaluated for accuracy by two independent judges, who agreed on $93 \%$ of the attempts and resolved their differences. Training attempts in the problem-oriented conditions of Experiment $2 \mathrm{~B}$ and target attempts in Experiment $2 \mathrm{~A}$ were scored by one of these judges.

\section{Results and Discussion}

\section{Percentage of Targets Solved}

Experiment 2A: Baseline. The percentages of correct solutions were submitted to an analysis of variance, with between-subject factors for mood and target type. Table 3 shows that performance was close to the level of performance on the training problems in Experiment 1, but much lower than performance on target problems. Dysphoric subjects solved $15 \%$ on average; the nondysphoric subjects solved $11 \%$. Moreover, none of the effects in this design was reliable $(F \mathrm{~s}<1.0)$.

Experiment 2B: Hints. The analysis of variance included between-subject factors for mood, training orientation, and type of target. Table 3 reports the means. The analysis revealed a reliable interaction of mood and training orientation $\left[F(1,88)=6.60, M S_{\mathrm{e}}=416.49\right] .^{2}$ The nondysphoric subjects solved more problems following memory-oriented training [ $81 \%$ vs. $55 \%$ after problem-oriented training; $F(1,88)=22.23$. According to the framework of transfer-appropriate processing, they were better equipped to use the hints they were given in the transfer phase, because their memory orientation during training was more compatible with the intentional retrieval processes that were invoked by the hints. Dysphoric subjects, however, performed similarly under the two training orientations $(70 \%$ after problem-oriented training vs. $74 \%$ after memory-oriented training; $F<1.0$ ).

As in Experiment 1, problem-oriented dysphoric subjects solved more target problems than did the nondysphoric subjects in that condition [70\% vs. $55 \%$, respectively; $F(1,88)=8.68]$. In contrast, dysphoric subjects solved slightly fewer problems following memoryoriented training than did the nondysphoric subjects ( $74 \%$ vs. $81 \%$, respectively), although the difference was not reliable $[F(1,88)=2.40]$.

This paradigm of memory-oriented training followed by instructions to recall is similar to the design of many depressive memory experiments: Subjects are asked to learn

Table 3

Mean Percentage of Targets Solved With Hints (Experiments 2A and 2B)

\begin{tabular}{lccc}
\hline & \multicolumn{3}{c}{ Type of Targets } \\
\cline { 2 - 4 } \multicolumn{1}{c}{ Training } & Neutral & Depressing & Overall \\
\hline No training (2A) & & & \\
$\quad$ Nondysphoric & 13.3 & 8.9 & 11.1 \\
$\quad \begin{array}{l}\text { Dysphoric } \\
\text { Problem oriented (2B) }\end{array}$ & 15.0 & 15.0 & 15.0 \\
$\quad$ Nondysphoric & & & \\
$\quad \begin{array}{l}\text { Dysphoric } \\
\text { Memory oriented (2B) }\end{array}$ & 61.4 & 48.6 & 55.0 \\
$\quad$ Nondysphoric & 70.0 & 70.0 & 70.0 \\
$\quad$ Dysphoric & 84.3 & 77.1 & 80.7 \\
\hline
\end{tabular}

Note-There were 9 nondysphoric and 8 dysphoric subjects in each condition of the no-training controls (Experiment $2 \mathrm{~A}$ ) and 14 nondysphoric and 10 dysphoric subjects in each experimental condition (Experiment 2B). 
something and then are tested for deliberate memory. Although depressive deficits are frequently found in such designs, one exception is provided by research that uses prose materials. Prose materials are sufficiently structured for effort- or initiative-related differences in initial learning to be unlikely to emerge (see Ellis \& Ashbrook, 1989; Hertel \& Hardin, 1990). Perhaps the prosodic nature of the problems we used was similarly engaging and benefited dysphoric subjects' attempts to recall the analogue. However, this interpretation, when combined with our previous interpretation of problem-oriented outcomes, assumes that the dysphoric subjects' approach to the hints depended on the type of training they had received. If they had tried to memorize, they used the hints; otherwise, they relied on the targets to remind them of the solutions. Given that the dysphoric subjects performed similarly following the two training orientations, a more parsimonious explanation is that they did not respond either to our instructions to memorize or to our instructions to remember the analogue. Regardless, the interesting outcome from the perspective of research on depression and cognition is that a depressive advantage in problem solving has been found and replicated. This outcome suggests that a lack of initiative can sometimes be beneficial.

\section{Percentage of Training Problems Solved in Experiment 2B}

The percentages of training problems solved in the problem-oriented conditions did not reliably differ according to mood or type of target; subjects solved $11 \%$, on average. As in Experiment 1, these percentages were reliably correlated with the percentages of targets solved $\left[\beta=.36 ; F(1,43)=6.58, M S_{\mathrm{e}}=382.10\right]$, but the analysis of covariance revealed only the reliable main effect of $\operatorname{mood} \operatorname{group}[F(1,43)=5.33]$.

\section{EXPERIMENT 3}

Experiment 2B replicated the dysphoric advantage obtained in Experiment 1 when problem-oriented subjects received hints about the appropriate analogy. The purpose of Experiment 3 was to replicate the finding from Experiment 1 that nondysphoric subjects trained with a problem orientation solved fewer problems when they were given hints than when they were not. Another purpose of Experiment 3 was to include all levels of training orientation and target instruction in the same experimental design. The previous designs each omitted the condition of memory-oriented training followed by nohint transfer. Needham and Begg (1991) had shown that this condition produced inferior performance on the transfer task (although better recall of training problems) relative to its problem-oriented counterpart. In line with their transfer-appropriate account, therefore, we expected that the nondysphoric subjects in this condition would solve fewer problems than would problem-oriented subjects in the no-hint condition and memory-oriented subjects who received hints. We did not attempt to recruit dysphoric participants, but we used both elaborated versions of the target problems (neutral and depressing) so that the design would be comparable to that of the preceding experiments.

\section{Method}

A total of 146 students participated in this experiment without having been screened with the BDI. The experimental data were not scored if the end-of-session BDI score was 9 or higher. There were 50 such subjects, but they were not evenly distributed across conditions, and the lack of multiple measures prevented a confident classification of dysphoria.

In the final sample of 96 subjects, equal numbers had been assigned to each combination of orientation during training (problemoriented vs. memory-oriented), instructions during transfer (hint vs. no hint), and type of target (neutral vs. depressing). In all except one combination, 8 of the 12 subjects were female ( 7 were female in the exception). Subjects participated in groups of 1 to 5 in sessions that varied according to the training orientation. The materials and procedures used in the training and transfer phases were identical to those used in the corresponding conditions of Experiments 1 and 2B. At the end of the sessions, we administered the $\mathrm{BDI}$ and mood questionnaire.

\section{Results and Discussion}

\section{Percentage of Targets Solved}

The percentages of correct solutions were submitted to an analysis of variance, with between-subject factors for training orientation, target instruction, and target type. Means are shown in Table 4 . The analysis revealed a reliable interaction of training orientation and target instruction $\left[F(1,88)=9.16, M S_{\mathrm{e}}=465.91\right]$. Apart from the main effect of orientation, all other effects in the overall design were nonreliable $(F \mathrm{~s}<1.0){ }^{3}$

As we found in Experiment 1, hints impaired the performance of problem-oriented subjects; on average, they solved $55 \%$ of the targets with hints but $68 \%$ without $[F(1,88)=4.02]$. In contrast, hints helped the performance of memory-oriented subjects, who solved $80 \%$ of targets with hints and only $66 \%$ without $[F(1,88)=5.17]$. These differences conformed to our expectations, as did the obviously reliable simple main effect of orientation when hints were provided.

The surprising outcome in this experiment was the failure to support the fourth prediction implied by the transferappropriate framework: Problem-oriented and memoryoriented subjects showed similar levels of spontaneous (no-hint) transfer (68\% vs. 66\%, respectively). This outcome constitutes a failure to replicate the results obtained in four experiments by Needham and Begg (1991). In searching for an explanation based on the differences in materials or procedures, we noted that the targets in this experiment were considerably longer than those used by Needham and Begg and were interspersed with fillers and rest intervals. It is difficult to imagine how the added length would work to the advantage of our memoryoriented subjects, but the longer retention intervals could deflate performance in the problem-oriented condition. 
Table 4

Mean Percentage of Targets Solved by Nondysphoric Subjects in Experiment 3

\begin{tabular}{lccc}
\hline \multicolumn{3}{c}{ Nondysphoric Subjects in Experiment 3} \\
\cline { 2 - 4 } Training & Neutral & Depressing & Overall \\
\hline Problem oriented & & & \\
$\quad$ No hints & 65.0 & 70.0 & 67.5 \\
$\quad$ Hints & 56.7 & 53.3 & 55.0 \\
Memory oriented & & & \\
$\quad$ No hints & 66.7 & 65.0 & 65.8 \\
$\quad$ Hints & 80.0 & 80.0 & 80.0 \\
\hline
\end{tabular}

Note- $n=12$.

\section{Percentage of Training Problems Solved}

These percentages did not reliably differ according to target instructions; the subjects who received problemoriented training solved $10 \%$ of the training problems, on average. The percentages of training and target problems solved were not reliably correlated $(r=.22)$.

\section{GENERAL DISCUSSION}

We began this research on problem solving by analogy with a focus on the nature of the memory component of spontaneous transfer. In considering the possibility of self-initiated, deliberate retrieval of the training analogue, we assumed that hints would benefit everyone but especially the dysphoric subjects, who typically show low initiative. In this regard, we found that hints improved performance only in the case of memory-oriented training (for nondysphoric subjects in Experiment 3). Following problem-oriented training, hints hurt the performance of nondysphoric subjects (Experiments 1 and 3 ) and had no reliable effect when subjects were dysphoric (Experiment 1). Following problem-oriented training, therefore, retrieval of the analogue's solution seems to have been accomplished by remindings during attempts to solve the target-a process that can be derailed by thematic hints to recall the analogue in preparation for the target. Moreover, this retrospective focus is apparently avoided in dysphoric mood states, as indicated by the dysphoric advantage in Experiments 1 and 2B. In the next sections, we relate these and other findings to the respective literatures on problem solving by analogy and cognitive processes in depression.

\section{Problem Solving by Analogy}

The literature on problem solving by analogy to prior experience presumably is based on performance by nondysphoric students, in the main. That literature documents many failures to show transfer unless hints have been given. An examination of the conditions for encountering the information to be transferred into the problem-solving phase reveals that they often involved direct provisions of solutions in the form of: (1) paired associates (Weisberg, DiCamillo, \& Phillips, 1978), (2) clue statements (Perfetto, Bransford, \& Franks, 1983), or (3) stories describing problems and their solutions (Catrambone \& Holyoak, 1989; Gick \& Holyoak,
1980). These conditions did not directly invoke a problem-oriented set during the training phase, but instead instructed for learning, comprehension, or other procedures incidental to both remembering and problem solving but helpful for remembering. Improvement from the subsequent provision of hints to remember is therefore not surprising, and our findings following memoryoriented training are consistent with this literature.

The literature on problem solving by analogy also contains evidence for spontaneous transfer (without hints). These findings have sometimes resulted from very simple manipulations of the form of the potential solutions given in the first or "training" phase. Perfetto et al.'s (1983) clue statements were transformed into puzzle or problem-oriented forms by Adams et al. (1988) and Lockhart et al. (1988). Compared with the statement forms, these conditions produced superior spontaneous transfer, according to predictions from the framework of transfer-appropriate processing. The puzzle and problemoriented forms induced a problem-solving set that was consistent with the approach to the target task and probably provided a basis for noticing the analogy. Similarly, Catrambone and Holyoak (1989) improved spontaneous transfer by including a problem to be solved along with structurally similar stories to be compared in the training phase. And, as previously discussed, Needham and Begg (1991) found higher levels of spontaneous transfer following problem-oriented training, as compared with memory-oriented training, when they held the form of the training material constant. Although we failed to replicate these results in Experiment 3, the other comparisons in all three experiments produced findings that were consistent with the transfer-appropriate framework. Noticing the analogy was best accomplished when the nature of the retrieval cues was appropriate to the training orientation. Retrieval cues in the form of the analogue's theme were successful when the analogue had been studied for later recall; when the structure of the training problem had been emphasized by problemoriented training, subjects were better off relying on cues inherent in the structure of the target problem.

Noticing the analogy is understood to be the key to successful spontaneous transfer, and it can be aided by surface similarities between the training and target problems (see Ross \& Kennedy, 1990). In this regard, a number of studies have shown evidence for spontaneous transfer by novice problem solvers when the training and target problems share many surface features (e.g., Holyoak \& Koh, 1987). Our training analogues were superficially dissimilar to the targets, but only in the broadest sense: The settings, objects, and types of "actors" differed. A reading of the examples in the appendix, however, suggests that the surface structures of the problems' descriptions were very much alike, at least in some sections. Even the use of particular strings of words might facilitate transfer (see Catrambone \& Holyoak, 1989; Stein et al., 1986). Such specificity is consistent with Ross and Kennedy's (1990) multicomponent view of problem solving by analogy and with instance-based 
theories of memory and concept learning (see Jacoby \& Brooks, 1984). Furthermore, as suggested by B. H. Ross (personal communication, September 6, 1993), providing a general cue concerning the theme of the analogue might be distracting when either superficial or structural similarities are sufficient to remind. Such cues possibly emphasize the dissimilarities rather than the similarities.

The detrimental effects of our hints might also depend on short intervals between training and transfer and on the between-subjects manipulation of hints. For example, after a 1-week delay and a cover story of separate experiments, Catrambone and Holyoak (1989) found that hints increased performance, even when training had been problem-oriented. Notably, they provided hints after subjects had attempted to solve without them, whereas our subjects had not been exposed to the target problem before being directed to think back to a particular training problem. In our case, a problem-oriented set might be harder to maintain.

Finally, the amount of time allotted for solution might also play a role. The 3-min limit did not appear to be instrumental in the effects obtained in Experiment 1, according to analyses of the number of incomplete solutions. However, the use of a strict limit, beginning in the training phase, might well establish a strategy that would favor spontaneous reminding and that would be disrupted by instructions to remember the analogue. On the other hand, the time was sufficient for memory-oriented subjects to use the hints quite effectively (Experiments $2 \mathrm{~B}$ and 3 ). Clearly, all of these issues deserve further investigation.

\section{Memory and Problem-Solving in Depressed Moods and States}

The finding of depression-related impairments in cognitive tasks is unfortunately ubiquitous. It ranges from reports of concentration, memory, and problem-solving difficulties in clinical interviews to similar evidence supplied by laboratory experiments (see Williams, Watts, MacLeod, \& Mathews, 1988). Some of that evidence suggests that impairments are more likely to be found when the cognitive task benefits from self-sustained attention. Nondepressed people are more likely to sustain attention in ways that ultimately benefit performance (see Hertel \& Rude, 1991). In contrast, our nondysphoric subjects appeared to sustain attention in detrimental ways--by taking the advice provided by the hints following problem-oriented training. Therefore, the finding of a depression- related advantage suggests that motivation or initiative is not always advantageous. Sometimes it's better to rely on the present situation for cues than to try to remember past experience.

The literature on depression and memory also documents deficits on tasks that benefit from effortful procedures employed in learning and remembering (see Ellis \& Ashbrook, 1988; Hasher \& Zacks, 1979; Hertel \& Milan, 1994; Williams et al., 1988). Certainly, the attempt to solve mathematical and logical problems often requires effortful procedures, but the benefit of prior experience with analogous problems need not derive from explicit reference to the past. Analogies can be invoked in less effortful and intentional ways. Roediger and McDermott (1992; Roediger, 1990) have set forth a similar argument regarding performance on implicit-memory tests, which are at least partly analogous to spontaneous transfer tasks in that they do not instruct subjects to remember but provide useful cues for retrieval. Moreover, the lack of a reliable dysphoric deficit in the no-hint condition of Experiment 1 is akin to results from several experiments on depression and implicit memory in which deficits were not obtained (Denny \& Hunt, 1992; Hertel \& Hardin, 1990; Watkins, Mathews, Williamson, \& Fuller, 1992). The trend toward poorer performance by our dysphoric subjects, however, might reflect impairments in other, more effortful components of transfer.

Those components might also have been impaired for dysphoric subjects who received memory-oriented training and hints to recall in Experiment 2B, because they showed a similar trend toward poorer performance. Although these weak and nonreliable trends toward deficits might merely reflect the low levels of depressive states in our subjects, the nature of the materials and the task might also play a role. Hertel and Rude (1991) have shown that even clinically depressed subjects can perform as well as nondepressed subjects when effortful procedures are guided by the design of the task. In particular, the interest value and story structure of our problems might have sustained subjects' attention and also prevented potential effects of distraction by irrelevant information contained in the elaborated target problems.

Success in solving a variety of problems, including our own, often depends on strategic processing. Dobson and Dobson (1981) found that depressed subjects were less strategic in discovering more difficult rules in concept learning and in applying them to new situations. In the domain of social problem solving, Marx, Williams, and Claridge (1992) also found that depressed subjects generated fewer and less effective strategies than did their nondepressed counterparts. In the present experiments, strategic processing was afforded in the problem-oriented conditions of the training phase and in the control conditions in Experiment 2A. In these cases, the trends favored the dysphoric subjects, but the generally low levels of performance prevent further speculation. The transfer phase also can be seen as a poor candidate for revealing deficits in self-initiated strategies, because subjects had been exposed to the correct method of solution during training. Instead of self-initiated strategies, prior learning was the basis of successful performance. In this regard, depressed subjects can be taught to use effective and effortful strategies in both cognitive and social problem-solving tasks (see Abramson, Alloy, \& Rosoff, 1981; Hertel \& Hardin, 1990; Nezu \& Perri, 1989). Therefore, our results are compatible with evidence that depressed moods do not impair performance that either primarily relies on implicit cues or results from the direct provision of effortful strategies.

In reviewing the outcomes of these experiments from the standpoint of mood-related differences, we think that 
it is important to notice that none of the experimental manipulations exerted reliable effects in the dysphoric groups. These outcomes might mean merely that the dysphoric subjects failed to follow instructions in either phase of the experiment (i.e., not just in the use of hints). This interpretation is, however, inconsistent with other features of our results. An effortful or uncooperative stance surely implies reduced attention to the experimenterprovided solutions and a lack of effort during the training and transfer phases, all of which would lead to reduced performance relative to that of the nondysphoric groups. In contrast, the possibility that instructions to use the hints, in particular, were not followed points to the advantage of a dysphoric mood under the appropriate conditions.

Finally, because many of our dysphoric subjects suffered only mild levels of depressed moods and none was formally diagnosed, it remains to be seen whether the results can be extended to clinically depressed samples. Although clinically depressed subjects might be impaired under conditions that depend on deliberate uses of memory and problem-solving procedures, we would nevertheless expect them to ignore hints for deliberate recall after problem-oriented training. Like Alloy and Abramson's (1979) depressed subjects who were "sadder but wiser," these depressed subjects should be sadder but more appropriate in their approach to problem solving by analogy.

\section{REFERENCES}

Abramson, L. Y., Alloy, L., \& Rosoff, R. (1981). Depression and the generation of complex hypotheses in the judgment of contingency. Behavior Therapy \& Research, 19, 35-45.

adams, L. T., Kasserman, J. E., Yearwood, A. A., Perfetto, G. A. Bransford, J. D., \& Franks, J. J. (1988). Memory access: The effects of fact-oriented versus problem-oriented acquisition. Memory \& Cognition, 16, 167-175.

ALlOY, L. B., \& ABRAmSON, L. Y. (1979). Judgment of contingency in depressed and nondepressed students: Sadder but wiser? Journal of Experimental Psychology: General, 108, 441-485.

Beck, A., Ward, C., Mendelson, M., Mock, J., \& Erbaugh, J. (1961). An inventory for measuring depression. Archives of General Psychiatry, 4, 561-571.

Catrambone, R., \& Holyoak, K. J. (1989). Overcoming contextual limitations on problem-solving transfer. Journal of Experimental Psychology: Learning, Memory, \& Cognition, 15, 1147-1156.

Denny, E. B., \& Hunt, R. R. (1992). Affective valence and memory in depression: Dissociation of recall and fragment completion. Journal of Abnormal Psychology, 101, 575-580.

DoBson, D. J. G., \& DoBson, K. S. (1981). Problem-solving strategies in depressed and nondepressed college students. Cognitive Therapy \& Research, 5, 237-249.

Ellis, H. C., \& AshBRooK, P. W. (1988). Resource allocation model of the effects of depressed mood states on memory. In K. Fiedler, \& J. Forgas (Eds.), Affect, cognition, and social behavior (pp. 25-43). Toronto: Hogrefe.

Ellis, H. C., \& Ashrrook, P. W. (1989). The "state" of mood and memory research: A selective review. Journal of Social Behavior \& Personality, 4, 1-21.

GiCK, M. L., \& Holyoak, K. J. (1980). Analogical problem solving. Cognitive Psychology, 12, 306-355.

Gick, M. L., \& McGARRY, S. J. (1992). Learning from mistakes: Inducing analogous solution failures to a source problem produces later successes in analogical transfer. Journal of Experimental Psychology: Learning, Memory, \& Cognition, 18, 623-639.

HASHER, L., \& ZACKS, R. T. (1979). Automatic and effortful processes in memory. Journal of Experimental Psychology: General, 108 356-388.

Hertel, P. T., \& Hardin, T. S. (1990). Remembering with and without awareness in a depressed mood: Evidence of deficits in initiative. Journal of Experimental Psychology: General, 119, 45-59.

Hertel, P. T., \& Milan, S. (1994). Depressive deficits in recognition: Dissociation of recollection and familiarity. Journal of Abnormal Psychology, 103, 736-742.

Hertel, P. T., \& Rude, S. S. (1991). Depressive deficits in memory: Focusing attention improves subsequent recall. Journal of Experimental Psychology: General, 120, 301-309.

НоцуоАК, K. J., \& Кон, K. (1987). Surface and structural similarity in analogical transfer. Memory \& Cognition, 15, 332-340.

INGRAM, R. E. (1990). Self-focused attention in clinical disorders: Review and a conceptual model. Psychological Bulletin, 107, 156-176.

JACOBY, L. L., \& BROOKs, L. R. (1984). Nonanalytic cognition: Memory, perception, and concept learning. In G. H. Bower (Ed.), The psychology of learning and motivation: Advances in research and theory (Vol. 18, pp. 1-47). New York: Academic Press.

Kendall, P. C., Hollon, S. D., Beck, A. T., Hammen, C. L., \& INGRAM, R. E. (1987). Issues and recommendations regarding use of the Beck Depression Inventory. Cognitive Therapy \& Research, 11, 289-299.

LockharT, R. S., LAMON, M., \& Gick, M. L. (1988). Conceptual transfer in simple insight problems. Memory \& Cognition, 16, 36-44.

Marx, E. M., Williams, J. M. G., \& Claridge, G. C. (1992). Depression and social problem solving. Journal of Abnormal Psychology, 101, 78-86.

MorRIS, C. D., BRANSFord, J. D., \& FranKs, J. J. (1977). Levels of processing versus transfer appropriate processing. Journal of Verbal Learning \& Verbal Behavior, 16, 519-533.

Мотт-Sмгтн, G. (1954). Mathematical puzzles for beginners and enthusiasts (2nd ed.). New York: Dover.

Needham, D. R., \& BegG, I. M. (1991). Problem-oriented training promotes spontaneous analogical transfer: Memory-oriented training promotes memory for training. Memory \& Cognition, 19, 543557.

Nezu, A. M., \& Perri, M. G. (1989). Social problem-solving therapy for unipolar depression: An initial dismantling investigation. Journal of Consulting \& Clinical Psychology, 57, 408-413.

Novick, L. R., \& HOLYOAK, K. J. (1991). Mathematical problem solving by analogy. Journal of Experimental Psychology: Learning, Memory, \& Cognition, 17, 398-415.

Perfetto, G. A., Bransford, J. D., \& Franks, J. J. (1983). Constraints on access in a problem solving context. Memory \& Cognition, 11, 24-31.

PhiLLIPS, H. (1961), My best puzzles in logic and reasoning. New York: Dover.

ROEDIGER, H. L., III (1990). Implicit memory: Retention without remembering. American Psychologist, 45, 1043-1056.

RoEDiger, H. L., III, \& MCDERMotT, K. B. (1992). Depression and implicit memory: A commentary. Journal of Abnormal Psychology, 101, 587-591.

Ross, B. H., \& KenNedy, P. T. (1990). Generalizing from the use of earlier examples in problem solving. Journal of Experimental Psychology: Learning, Memory, \& Cognition, 16, 42-55.

SeiberT, P. S., \& Ellis, H. C. (1991). Irrelevant thoughts, emotional mood states, and cognitive task performance. Memory \& Cognition, $19,507-513$

Stein, B. S., Way, K. R., Benningfield, S. E., \& Hedgecough, C. A. (1986). Constraints on spontaneous transfer in problem-solving tasks. Memory \& Cognition, 14, 432-441.

Watkins, P. C., Mathews, A., Williamson, D. A., \& Fuller, R. D. (1992). Mood-congruent memory in depression: Emotional priming or elaboration? Journal of Abnormal Psychology, 101, 581-586.

Weisberg, R., DiCamillo, M., \& Phillips, D. (1978). Transferring old associations to new situations: A nonautomatic process. Journal of Verbal Learning \& Verbal Behavior, 17, 219-228.

Williams, J. M. G., Watts, F. N., MacLeod, C., \& Mathews, A. (1988). Cognitive psychology and emotional disorders. New York: Wiley. 


\section{NOTES}

1. The distributions of correct solutions to the individual targets each showed the pattern found in the overall interaction. Furthermore, an analysis of variance with repeated measures for target number failed to reveal reliable interactions of that factor with mood or instructions

2. The pattern in the overall interaction was also seen in the distributions of correct responses for each target, and the inclusion of target number as a within-subjects factor in the overall design failed to reveal reliable interactions with this factor.

3. The pattern of means contributing to the interaction of orientation and instruction was also present in the distribution of correct responses to Targets 1,3 , and 4 . Targets 2 and 5 showed a pattern more aligned to the main effect of orientation. By including a within-subjects factor for target number in the analysis of variance, we found no reliable interactions involving this factor.

\section{APPENDIX}

\section{Sample Training Problem: Two-String (Needham \& Begg, 1991)}

An adventurous explorer traveling through the jungles of Africa decided to stop for the night. Since the jungle he was exploring was full of snakes, he decided to sleep in a hammocklike device suspended over a babbling brook. He began unfolding the blanket that would serve as the base for the hammock. When he finished this, the explorer grabbed two vines hanging down and tied them together. This served as support for one end of the blanket. However, the two vines that were to support the other end presented some difficulty. When the explorer grabbed the end of one vine, it was impossible for him to grasp the end of the other vine at the same time. The two vines simply could not be knotted together in this way. The explorer though he would have to give up and move camp elsewhere because these two vines from above could not be knotted together. Suddenly, an idea struck the explorer and he was able to knot together the two vines. How?

\section{Sample Target Problem}

\section{Simple Version}

Before [the dance], organizers were hurriedly trying to decorate the hall. Everything was nearly ready, and it was about ten minutes before the guests were scheduled to arrive. [Chris] was decorating the walls and ceiling with balloons and party streamers made out of ribbon. He had nearly completed a fancy decoration pattern when he noticed two final pieces of ribbon were left dangling from the tiled ceiling above. He had planned to knot these two final pieces of ribbon together in order to attach balloons to them. However, when he grabbed the end of the green ribbon, he was unable to grasp the end of the blue ribbon at the same time. The ribbons could simply not be knotted together in this way. Since everyone had momentarily left the room, [Chris] thought that he would have to abandon this bit of decoration altogether. Suddenly, an idea struck him, and he was able to knot together these two ribbons. How?

(The brackets denote substitutions in the original problem from Needham \& Begg, 1991.)

\section{Neutral Elaborated Version}

Before the dance, organizers were hurriedly trying to decorate the hall. Everything was nearly ready, and it was about ten minutes before the guests were scheduled to arrive. Chris was decorating the walls and ceiling with balloons and party streamers made out of ribbon. Chris was on the decoration committee for the prom. He was one of those kids who was always pitching in as a way of keeping up school spirit and a sense of community, and this committee had worked out well; everyone had pitched in and done a good job without too much hassle. He had nearly completed a fancy decoration pattern when he noticed two final pieces of ribbon were left dangling from the tiled ceiling above. Time was running out, and Chris needed some time to shower and change before picking up his friend, who understood that he would be a bit late. He had planned to knot these two final pieces of ribbon together in order to attach balloons to them and then go home to change. However, when he grabbed the end of the green ribbon, he was unable to grasp the end of the blue ribbon at the same time; his arms weren't long enough. The ribbons could simply not be knotted together in this way. Since everyone had left to dress for the dance, Chris thought that he would have to abandon this bit of decoration altogether. His friend was expecting him to be at her house in about forty-five minutes, and she lived fifteen minutes away from his house. Suddenly, an idea struck him, and he was able to knot together these two ribbons. How?

\section{Depressing Elaborated Version}

Before the dance, organizers were hurriedly trying to decorate the hall. Everything was nearly ready, and it was about ten minutes before the guests were scheduled to arrive. Chris was decorating the walls and ceiling with balloons and party streamers made out of ribbon. Chris was on the decoration committee for the prom. He was one of those kids who was always on committees as a way of trying to be involved and have a social life, but this committee had worked out like all the others; no one paid any attention to him, except to give him all the unpleasant jobs. He had nearly completed a fancy decoration pattern when he noticed two final pieces of ribbon were left dangling from the tiled ceiling above. Time was running out, and Chris was afraid that the other seniors would arrive for the prom and see him standing there in his school clothes. He had planned to knot these two final pieces of ribbon together in order to attach balloons to them and cover up his mistake. However, when he grabbed the end of the green ribbon, he was unable to grasp the end of the blue ribbon at the same time, no matter how hard he tried. The ribbons could simply not be knotted together in this way. Since everyone had left to dress for the dance, Chris thought that he would have to abandon this bit of decoration altogether. His mother was expecting him to be home to take care of his little brother while she went to work. Suddenly, an idea struck him, and he was able to knot together these two ribbons. How?

\section{Scoring Criteria}

To be scored correct, the attempt must mention the following: (a) Tie something to the end of one of the ribbons. (b) Swing the ribbon with the object tied to it. (c) Catch the swinging ribbon and tie the two together.

(Manuscript received June 20, 1994; revision accepted for publication January $16,1995$. 